\title{
PENSAMIENTO COMPLEJO: UNA REVISIÓN SISTEMÁTICA DE ARTÍCULOS CIENTÍFICOS INDEXADOS EN SCOPUS 2016-2019
}

\section{Complex Thinking: A Systematic Review of Scientific Articles Indexed in Scopus 2016-2019}

\author{
Jorge Alberto Flores Morales \\ Universidad Femenina Sagrado Corazón. jorgefloresm@unife.pe \\ ID ORCID: 0000-0002-3678-5511
}

\section{RESUMEN:}

Objetivo: Identificar, revisar sistemáticamente y resumir la mejor evidencia científica disponible sobre producción de artículos originales y empíricos sobre el pensamiento complejo. entre los años 2016-2019.

Método: La búsqueda se realizó utilizando la base de datos Scopus en acceso abierto, utilizando referencias de estudios primarios y secundarios que se encontraron. La búsqueda se realizó en base al término "pensamiento complejo" o afines descartándose aquellos que no hagan referencia a la propuesta de Edgar Morin.

El resultado fue de 11 artículos de trabajo, de estos se han excluido aquellos que no cumplían con el objetivo de estudio.

\section{PALABRAS CLAVE:}

Pensamiento complejo, Edgar Morin, paradigmas, multidimensional.

\begin{abstract}
Objective: To identify, systematically review and summarize the best available scientific evidence on the production of original and empirical articles on complex thinking. between the years 2016-2019.
\end{abstract}

Method: The search was carried out using the Scopus database in open access, using references of the primary and secondary studies that were found. The search was carried out on the basis of the term "complex thought" or related, discarding those that do not refer to Edgar Morin's proposal.

The result was 11 articles of work, of these those that did not fulfill the objective of the study have been excluded.

\section{KEYWORDS:}

Complex thinking, Edgar Morin, paradigms, multidimensional. 


\section{INTRODUCCIÓN}

El pensamiento complejo implica enfrentar la realidad, que se nos presenta múltiple y sorprendente, de una manera estratégica. Al enfrentarnos a esta realidad compleja debemos hacerla de manera reflexiva.

El mundo es un todo indisociable a pesar de sus diversas aristas que la componen y frente a ella buscamos relacionarnos a través de certezas sabiendo que no todo resulte diáfano; obliga entonces a buscar un abordaje multidisciplinario partiendo de un análisis de la realidad que implica tener en cuenta no solo el momento presente sino también su historia y su devenir. Es así que en el prólogo de introducción de su obra Morin (1994 p.23) señala:

Nunca pude, a lo largo de toda mi vida, resignarme al haber parcelarizado, nunca pude aislar un objeto de estudio de su contexto, de sus antecedentes, de su devenir. He aspirado siempre a un pensamiento multidimensional. Nunca he podido eliminar la contradicción interior. Siempre he sentido que las verdades profundas, antagonistas las unas de las otras, eran para mí complementarias, sin dejar de ser antagonistas. Nunca he querido reducir a la fuerza la incertidumbre y la ambigüedad.

El pensamiento complejo plantea una mirada reflexiva entorno la heterogeneidad, la interacción y el azar, que me lleva a interconectar toda la realidad u objeto del conocimiento por el cual éste no es un ente aislado, sino que está inserto en un sistema relacional. En palabras de Morin un sistema es: "Una interrelación de elementos que constituyen una entidad o unidad global. Tal definición comporta dos caracteres principales, el primero es la interrelación de los elementos, el segundo es la unidad global constituida por estos elementos en interrelación." (Morin, 1993, p.123)

Por ello, hay que diferenciar el término complejo que usa la ciencia clásica al insistir más en la divisibilidad o en su totalidad como completud o pensar que la complejidad elimina la simplicidad, estas ilusiones sobre la complejidad lo llamará Morin, Complejidad restringida.

Se puede señalar algunos principios del pensamiento complejo como la "dialógica" donde se advierte que la utilización de más de un significado de un término en un mismo enunciado no ayuda a la construcción del conocimiento por el cual se ha de buscar la complementación de las partes en función a un mejor resultado que intervenciones individuales; la "recursividad" que expresa la ambivalencia de los elementos; "Hologramático" que ve la construcción del conocimiento donde el TODO se encuentre en las partes y viceversa; la autorganización donde los fenómenos no son hechos consumado sino que estos van cambiando y reorganizándose tendente a un fin o meta en relación con el medio; "Metanoia" en vista que el conocimiento sea dinámico y abierto la realidad debe ser apreciada desde diferentes posturas o perspectiva que en esta dinámica creativa genera innovación y la "Metacognición" que nos pone frente a nuestro propio desempeño al contacto con la realidad.

Según Morin, la falta de la "visión global" (producto del pensamiento 
complejo) implica la pérdida de conciencia respecto de nuestra verdadera condición humana, es decir, nuestra condición de personas que habitan un mundo y que mantienen lazos afectivos, espirituales, sociales, económicos, etc., con otros.

Para Morin, nuestra vida supone una multiplicidad de relaciones (así como una diversidad de ámbitos en los que se desenvuelve). Tomar conciencia de esa multiplicidad es lo que nos permite desarrollarnos como sujetos humanos (en un sentido integral del término) y no sólo como simples objetos. Así, una visión diferente del mundo (basada en el pensamiento complejo) supone un cambio en la manera en que afrontamos la vida.

\section{METODOLOGÍA}

\section{El diseño y el sitio del estudio}

Esta fue una revisión sistemática realizada entre los días 20 y 21 de agosto de 2020 a partir de la base de datos de Scopus.

\section{Criterios de elegibilidad}

Tipos de artículo: Se incluyeron artículos originales y empíricos que lleven en el título en su producción "pensamiento o sistema complejo" o afines descartándose títulos que no tengan como referente la interpretación de Edgar Morin.

\section{Selección de estudios}

El proceso de selección de los estudios fue realizado por dos revisores independientes, y cualquier divergencia fue resuelta por un tercer revisor. Los estudios se seleccionaron en dos etapas. El primer paso consistió en revisar los títulos y resúmenes de las referencias encontradas con nuestra estrategia de búsqueda; se seleccionaron los estudios potencialmente elegibles. El segundo paso consistió en revisar el texto completo de los estudios preseleccionados para confirmar su elegibilidad.

\section{Tipos de estudios}

Considerando el número limitado de estudios sobre pensamiento complejo, el objetivo de esta breve revisión es mapear los trabajos existentes sobre el tema e identificar aquellos que son artículos originales y empíricos separados en dos tablas para su mejor estudio.

\section{RESULTADOS}

\section{Características de los estudios incluidos}

Las 11 referencias seleccionadas fueron leídas para confirmar la elegibilidad (segundo paso). En la primera tabla se seleccionaron 6 artículos originales donde en sus resúmenes claramente señalan objetivo, método y conclusiones entre otros para su elegibilidad cuyos años se extiende desde 2016 al 2019. En la segunda tabla son 5 artículos empíricos donde se identifica básicamente el objetivo o intencionalidad del artículo, la situación de estudio y la conclusión arribada en el estudio que van desde los años 2017 al 2019.

Los resultados de los 11 estudios se presentan a continuación de manera concisa y descriptiva, ya que la naturaleza de estos estudios no permite ningún otro tipo de análisis. 
Tabla 1 Artículos originales

\begin{tabular}{|c|c|c|c|c|}
\hline $\begin{array}{c}\text { Autor(es), Título y } \\
\text { año }\end{array}$ & $\begin{array}{l}\text { Idioma } \\
\text { original }\end{array}$ & Objetivo & Métodos & Conclusiones \\
\hline $\begin{array}{c}\text { Costa, V. T., E } \\
\text { Meirelles, B. H. S. } \\
\text { (2019). Adhesión al } \\
\text { tratamiento de los } \\
\text { adultos jóvenes que } \\
\text { viven con VIH/SIDA, } \\
\text { según la óptica del } \\
\text { pensamiento com- } \\
\text { plejo. } \\
\text { https://doi.or- } \\
\text { g/10.1590/1980- } \\
\text { 265X-TCE-2017-0016 }\end{array}$ & Portugués & $\begin{array}{c}\text { Comprender } \\
\text { la adherencia } \\
\text { al tratamiento } \\
\text { de los adultos } \\
\text { jóvenes con } \\
\text { VIH / SIDA } \\
\text { atendidos en } \\
\text { un servicio } \\
\text { de atención } \\
\text { especializada } \\
\text { desde la pers- } \\
\text { pectiva del } \\
\text { pensamiento } \\
\text { complejo }\end{array}$ & $\begin{array}{c}\text { Estudio } \\
\text { cualitativo, } \\
\text { con enfoque } \\
\text { de teoría } \\
\text { fundamenta- } \\
\text { da en datos. }\end{array}$ & $\begin{array}{l}\text { Se consideró } \\
\text { que, en vista del } \\
\text { fenómeno com- } \\
\text { plejo y cambian- } \\
\text { te, la adhesión al } \\
\text { tratamiento de } \\
\text { los adultos } \\
\text { jóvenes con VIH/ } \\
\text { SIDA debe ser } \\
\text { comprendida y } \\
\text { manejada por } \\
\text { los profesionales } \\
\text { de la salud como } \\
\text { un todo } \\
\text { complejo. }\end{array}$ \\
\hline $\begin{array}{c}\text { Medeiros, S., E } \\
\text { Santos, V. (2019). } \\
\text { Sistema de medi- } \\
\text { cación y seguridad } \\
\text { del paciente a la luz } \\
\text { de la teoría de la } \\
\text { complejidad. ISSN: } \\
08640319\end{array}$ & Portugués & $\begin{array}{c}\text { Reflexionar } \\
\text { sobre la } \\
\text { complejidad } \\
\text { del sistema } \\
\text { de medica- } \\
\text { ción y su } \\
\text { interfaz con } \\
\text { la seguridad } \\
\text { del paciente } \\
\text { a partir de los } \\
\text { conceptos de } \\
\text { la teoría de } \\
\text { Edgar Morin. }\end{array}$ & $\begin{array}{c}\text { Estudio } \\
\text { reflexivo } \\
\text { desarrolla- } \\
\text { do a partir } \\
\text { de lecturas } \\
\text { correlacio- } \\
\text { nadas con el } \\
\text { área temáti- } \\
\text { ca, mediante } \\
\text { artículos } \\
\text { científicos } \\
\text { disponibles } \\
\text { en las bases } \\
\text { de datos } \\
\text { Biblioteca } \\
\text { Electrónica } \\
\text { Científica } \\
\text { en Línea y } \\
\text { Biblioteca } \\
\text { Virtual en } \\
\text { Salud. La } \\
\text { selección de } \\
\text { los materia } \\
\text { les ocurrió } \\
\text { en agosto de } \\
2016 .\end{array}$ & $\begin{array}{l}\text { La articulación } \\
\text { entre el pa- } \\
\text { radigma de la } \\
\text { complejidad y } \\
\text { el sistema de } \\
\text { medicación nos } \\
\text { permite vislum- } \\
\text { brar oportunida- } \\
\text { des de mejora } \\
\text { en la calidad de } \\
\text { la atención segu- } \\
\text { ra. Sin embargo, } \\
\text { para una mejor } \\
\text { comprensión de } \\
\text { estos sistemas, } \\
\text { es necesario } \\
\text { desarrollar más } \\
\text { investigaciones } \\
\text { para contribuir } \\
\text { de manera efec- } \\
\text { tiva a la aten- } \\
\text { ción médica del } \\
\text { paciente. }\end{array}$ \\
\hline
\end{tabular}




\begin{tabular}{|c|c|c|c|c|}
\hline $\begin{array}{c}\text { Cruz, R. A., Araujo, } \\
\text { E. L., Nascimen- } \\
\text { to, N. M., Lima, } \\
\text { R. J., França, J. R., } \\
\text { E Oliveira, J. D. } \\
\text { (2017). Reflexiones } \\
\text { a la luz de la Teoría } \\
\text { de la Complejidad } \\
\text { y la formación de } \\
\text { enfermeras. Revista } \\
\text { Brasileira De En- } \\
\text { fermagem, 70(1), } \\
\text { 236-239. https:// } \\
\text { doi:10.1590/0034- } \\
\text { 7167-2016 0239 }\end{array}$ & Portugués & $\begin{array}{l}\text { Reflexionar } \\
\text { sobre la } \\
\text { formación en } \\
\text { Enfermería } \\
\text { teniendo en } \\
\text { consideración } \\
\text { los principios } \\
\text { del pensar } \\
\text { complejo } \\
\text { propuesto } \\
\text { por Morin. }\end{array}$ & $\begin{array}{c}\text { Reflexión } \\
\text { fundamen- } \\
\text { tada en los } \\
\text { principios de } \\
\text { la Teoría de } \\
\text { la Compleji- } \\
\text { dad de Edgar } \\
\text { Morin. }\end{array}$ & $\begin{array}{l}\text { El cuidado de } \\
\text { Enfermería ha } \\
\text { sido basado en } \\
\text { una asistencia } \\
\text { reduccionista, } \\
\text { reflexionando el } \\
\text { modelo carte- } \\
\text { siano. Así, la } \\
\text { formación del } \\
\text { enfermero busca } \\
\text { contemplar } \\
\text { saberes y } \\
\text { experiencias } \\
\text { compartidas de } \\
\text { manera que no } \\
\text { exista el domi- } \\
\text { nio de ninguna } \\
\text { disciplina sobre } \\
\text { las otras, de nin- } \\
\text { gún profesional } \\
\text { sobre otro, } \\
\text { aceptando las } \\
\text { singularidades } \\
\text { tanto de los } \\
\text { profesionales } \\
\text { como del propio } \\
\text { cliente/paciente. }\end{array}$ \\
\hline $\begin{array}{l}\text { Ferreira, A. C. Z., } \\
\text { Brusamarello, T., Ca- } \\
\text { pistrano, F. C., Marin, } \\
\text { M. J. S., E Maftum, } \\
\text { M. A. (2017).La } \\
\text { vivencia del por- } \\
\text { tador de trastorno } \\
\text { mental en el uso de } \\
\text { psicofármacos en la } \\
\text { perspectiva del pen- } \\
\text { samiento complejo. } \\
\text { Doi: } 10.1590 \text { / } 0104- \\
\text { 07072017001000016 }\end{array}$ & Portugués & $\begin{array}{l}\text { Comprender } \\
\text { cómo los } \\
\text { pacientes con } \\
\text { trastornos } \\
\text { mentales } \\
\text { experimentan } \\
\text { el uso de me- } \\
\text { dicamentos } \\
\text { psicotrópicos }\end{array}$ & $\begin{array}{c}\text { Investigación } \\
\text { cualitativa, } \\
\text { exploratoria } \\
\text { y descrip- } \\
\text { tiva, que } \\
\text { utilizó como } \\
\text { referencia } \\
\text { teórica el } \\
\text { pensamiento } \\
\text { complejo de } \\
\text { Edgar Morin. }\end{array}$ & $\begin{array}{l}\text { Las vivencias de } \\
\text { los pacientes } \\
\text { con trastorno } \\
\text { mental en el uso } \\
\text { de medicamen- } \\
\text { tos psicotrópi- } \\
\text { cos es un pro- } \\
\text { ceso dinámico } \\
\text { y complejo que } \\
\text { engloba la multi- } \\
\text { dimensionalidad } \\
\text { que involucra al } \\
\text { ser humano y su } \\
\text { tratamiento. }\end{array}$ \\
\hline
\end{tabular}


Díaz, O. H., E Arboledad, A. P. (2016). Educación ciudadana en el marco del pensamiento complejo https:// doi.org/10.22507/rli. v13n2al2
Portugués convivencia y la ciudadanía. Materiales y métodos. Se realizó una investigación cualitativa y se aplicó un método de investigación basado en la etnometodología.

Copelli, F. H. S., de Oliveira, R. J. T., de Oliveira, C. M. S., Meirelles, B. H. S., de Mello, A. L. S. F., E Magalhães, A. L. P. (2016).El pensamiento complejo y sus repercusiones en la gestión en enfermería y salud http://dx.doi. org/10.5294/ aqui.2016.16.4.8.
Portugués

\section{Evidenciar} conocimientos científicos sobre la teoría de la complejidad en publicaciones científicas en enfermería y salud. sobre gestión
Se realizó una inves-

tigación

cualitativa, y

se aplicó un método de investigación sustentado en la etnometolodogía

Se trata de una revisión integradora de las bases de datos LILACS, BDENF y PubMed. Incluye diez artículos publicados entre $2004 \mathrm{y}$ 2013
Es importante realizar una reflexión pedagógica sobre el conocimiento y el desarrollo de la ciudadanía en el sistema educativo colombiano, dentro de un marco de pensamiento complejo, abordando temas como la evaluación, el rol de los estudiantes, el rol del profesor, el currículo y la investigación.

El pensamiento complejo es un paradigma relacionado a la integralidad, multidisciplinariedad, atención a las redes de apoyo y articulación de los saberes. Se ha usado como modelo de gestión en organizaciones vivas e imprevisibles para promover la organización de los sistemas complejos adaptativos. 
El primer artículo, presentado por Costa, V. T., E Meirelles, B. H. S. (2019). corresponde a una investigación original, realizada en Brasil. Este estudio tuvo como muestra a 12 adultos jóvenes con edades de 15 a 24 años, con VIH/SIDA, nueve profesionales de la salud y cuatro madres. El método que utilizaron estudio cualitativo, con el enfoque de la teoría fundamentada en los datos.

Los resultados obtenidos en este estudio indican que la adhesión al tratamiento de adultos jóvenes con VIH/ SIDA se comprendió como un fenómeno complejo, dinámico, multifactorial y en constante cambio. Tal proceso implica varios aspectos, entre ellos el miedo a sufrir, a la muerte física y social, a la discriminación y al estigma. Se constató que, pese a estas dificultades, los adultos jóvenes deciden seguir sus tratamientos en búsqueda de normalizar su salud, de una vida prolongada y común como los demás jóvenes que no viven con VIH/ SIDA. Sobre la base de los resultados, se sugiere que se realicen más estudios con esta población para comprender este fenómeno complejo y cambiante de adherencia al tratamiento. El ser joven con VIH / SIDA se refiere a un Ser de impulso y deseo que forma parte de un todo social; un ser trinitario constituido por su individualidad, su especie biológica $y$, al mismo tiempo, un ser social. Por tanto, el ser joven con VIH / SIDA es un Ser Complejo. Para eso, es necesario reconocer lo biológico, organizacional, político, individual, aspectos culturales y sociales que permean la vida de los adultos jóvenes con VIH / SIDA, entre ellos la adherencia al tratamiento.

La importancia/relevancia de este estudio fue relacionar la propuesta de pensamiento complejo de Morin con la presencia de la enfermedad del sida.
Así la adherencia al tratamiento de los adultos jóvenes debe ser entendida y manejada por profesionales de la salud en su conjunto complejo. Para eso, es necesario dejar la lógica del paradigma reduccionista y avanzar hacia una lógica compleja, es decir, es necesario reformar el pensamiento, (re)pensar y (re) construir conductas, acciones y estrategias diarias dirigidas a la adherencia al tratamiento de adultos jóvenes viviendo con VIH / SIDA, incluyendo la adopción de acciones de salud para más allá de la clínica, es decir, acciones que tengan en cuenta la singularidad de los adultos jóvenes ante la complejidad que es la adherencia al tratamiento del VIH / SIDA

El segundo artículo presentado por Medeiros, S., E Santos, V. (2019). corresponde a una investigación original, realizada en Brasil. El método que se utilizó en este trabajo es de enfoque cualitativo. Se trata de un estudio reflexivo, desarrollado como producto de la disciplina Filosofía y Epistemología de la Ciencia. Esta producción reflexiva se construyó a partir de lecturas correlacionadas con el área temática, a través de artículos científicos disponibles en las bases de datos electrónicas Scientific Eletronic Library Online (SciELO) y Virtual Health Library (BVS).

Los resultados indicaron que la teoría de Edgar Morin aportó reflexiones sobre la complejidad del sistema de medicación y su interfaz con la seguridad del paciente a partir de la conceptualización de los principios que constituyen el pensamiento complejo. Al defender una reforma del pensamiento, a través de la inseparabilidad de los fenómenos, se entiende su abordaje con aspectos importantes que involucran a este sistema, los cuales deben ser articulados 
e interconectados para prevenir la ocurrencia de EA y garantizar la seguridad de los pacientes.

El análisis de los principios de la teoría de la complejidad y su interfaz con SP y el sistema de medicación permitió reflexiones considerables sobre el tema. Este proceso requiere la articulación entre todas sus etapas, así como la asociación de la información inherente a cada fase, lo que caracteriza su complejidad. En este sentido, fortalecer esta conexión permitirá la perspectiva de evitar errores con la medicación y favorecerá la SP en las instituciones de salud, basada en la calidad de la atención

La importancia/relevancia de este estudio fue que en medio la presencia de EA relacionados con la medicación requiere que los errores se compartan entre el equipo. En esta perspectiva, los profesionales, basados en conocimientos interconectados, buscan adoptar nuevas estrategias para reducir estos eventos. En vista de lo anterior, el principio de recursividad propone la autoorganización y aborda que se acepte el desorden para que el sistema pueda reorganizarse y generar un nuevo orden. Por tanto, es a través de la identificación del EA por parte del equipo (trastorno), a través de las notificaciones, que se elaborarán nuevas medidas, con el intercambio de información, con el fin de buscar una reorganización en el sistema de medicación y contribuir al SP. (30)

Por tanto, es inevitable que la aparición de EA, de difícil comprensión y detección, se produzca en medio de una compleja cadena en el proceso de medicación. Por tanto, en su ocurrencia, lo más importante no es señalar quién cometió el fallo, sino cómo y por qué falló el sistema de defensa
El tercer artículo presentado por Cruz, R. A., Araujo, E. L., Nascimento, N. M., Lima, R. J., França, J. R., E Oliveira, J. D. (2017). corresponde a una investigación original, realizada en Brasil.

Los resultados indicaron que la aplicación de la complejidad en la educación propone una educación emancipadora basada en el cuestionamiento y la transformación social. Incluye la formación de enfermeros que tienen como característica de su trabajo la interacción con los demás. Si es necesario preparar al aprendiz para desarrollar acciones críticas y reflexivas y acciones capaces de superar la fragmentación y la linealidad del conocimiento. Al utilizar el pensamiento complejo, los profesionales de enfermería recurren a la reconfiguración del conocimiento disciplinar, donde los conocimientos y experiencias comienzan a reflejarse y compartirse de manera que no haya dominio de ninguna disciplina sobre las demás, de ningún profesional sobre el otro. , con el fin de brindar una atención ampliada, segura y eficaz, respetando y aceptando las singularidades tanto de los profesionales asistenciales como del propio cliente / paciente como participante activo en el proceso.

La importancia/relevancia de este estudio fue que el cuidado de enfermeros no puede concebirse como una acción reduccionista y simplificadora, sino como una construcción singular, que implica interacciones, reflexiones y autoconocimientos. Enfermería, a la hora de construir una atención ampliada, ha buscado contemplar constantemente acción-reflexiónacción. Teniendo en cuenta que la construcción del conocimiento no pasa por la suma de conocimientos, sino por la transformación y organización de lo aprendido, la complejidad explica que 
la búsqueda de la unidad muchas veces lleva al ser humano a intentar igualar las partes borrando sus particularidades. Sin embargo, las características de cada parte deben conservarse para que haya una comprensión completa del todo. Los resultados muestran que, durante la graduación, es necesario prestar atención a cómo se abordan los contenidos, para que las sucesivas aproximaciones no sean solo un cúmulo de nuevos temas

El cuarto artículo, presentado por Ferreira, A. C. Z., Brusamarello, T., Capistrano, F. C., Marin, M. J. S., E Maftum, M. A. (2017). corresponde a una investigación original, realizada en Brasil. Este estudio tuvo como muestra a 26 portadores de trastorno mental de 18 años a más con la prescripción de psicofármacos y fueron sometidos al análisis categorial temático por medio de entrevista semiestructurda. El método estaba basado en la investigación cualitativa, exploratoria y descriptiva que utilizó como referencial teórico el pensamiento complejo de Edgar Morin.

Los resultados obtenidos en este estudio indican que emergió una categoría central: "La vivencia compleja del portador de trastorno mental en el uso de psicofármacos", que muestra las interrelaciones entre las categorías temáticas: vivenciando los efectos de los psicofármacos; utilizando los psicofármacos de modo irregular; identificando facilidades y dificultades en el uso de psicofármacos; y desarrollando estrategias para mantenimiento del uso regular de psicofármacos. Sobre la base de los resultados, se sugiere que destacar que obtenido los resultados estas pueden favorecer la interrupción de la simplificación de prácticas e intervenciones fragmentadas que todavía predominan en la atención de la salud mental, por una la concepción multidimensional que involucra al ser humano y su tratamiento.

La importancia/relevancia de este estudio fue que permitió comprender que la experiencia de los pacientes con trastornos mentales es un proceso dinámico y complejo. Cuando se someten a terapia medicinal aquellos con trastornos mentales. La experiencia de los pacientes con trastornos mentales que utilizan psicotrópicos permanece constantemente experimentando los efectos de la medicación psicotrópica expresados por los efectos positivos y negativos con sentimiento de ambivalencia. Estas dimensiones emergentes cambian constantemente y están estrechamente relacionados e influenciados unos por otros, constituyendo así la experiencia compleja de la persona con trastorno mental en el uso de medicamentos psicotrópicos.

El quinto artículo, presentado por Díaz, O. H., E Arboledad, A. P. (2016). corresponde a una investigación original, realizada en Colombia. Este estudio se basó en los procesos de educación con énfasis en la formación en convivencia y ciudadanía. El método estaba basado en el enfoque cualitativo. Se realizó una investigación cualitativa, y se aplicó un método de investigación sustentado en la etnometolodogía. Los resultados obtenidos en este estudio versan sobre la relación que se da a partir del pensamiento complejo y la educación ciudadana, estableciendo un desarrollo teórico que se sustenta en las categorías de pensamiento complejo y ciudadanía, para terminar en la articulación en el campo educativo de estos dos temas y las posibles repercusiones que en el nivel pedagógico se puedan presentar. Sobre la base de los resultados, se 
sugiere que los modelos pedagógicos en educación existentes preservan la distancia entre maestros y estudiantes de la reflexión crítica de la realidad circundante, lo cual es un gran error, ya que, como lo sostiene Correa (2012), la pedagogía debe asumirse desde una postura crítica de auto-investigación que no se centre en una relación lineal de sujeto-objeto, sino en un diálogo transformador entre persona-persona, conscientes del reconocimiento de la subjetividad del otro sujeto, con la posibilidad de influir en la transformación individual y colectiva. Por otra parte, en lo relacionado con los educadores, estos deberán ser capaces de tomar posturas críticas y complejas, ya que eso permite que fomente en los estudiantes el análisis de la realidad social que repercuten en la formación de una conciencia crítica para la transformación social, generando en ellos la formulación de preguntas sobre su contexto, el cual tenga en cuenta la multiplicidad de manifestaciones de los asuntos humanos.

La importancia/relevancia de este estudio fue que la reflexión pedagógica sobre el reconocimiento y desarrollo de la ciudadanía en el sistema educativo en Colombia a partir del paradigma complejo, abordando temas como la evaluación, el rol de estudiante y de profesor, el currículo y la investigación

El sexto artículo, presentado por Copelli, F. H. S., de Oliveira, R. J. T., de Oliveira, C. M. S., Meirelles, B. H. S., de Mello, A. L. S. F., E Magalhães, A. L. P. (2016), corresponde a una investigación original, realizada en Brasil. Este estudio tuvo como muestra a 12 adultos jóvenes con edades de 15 a 24 años, con VIH/ SIDA, nueve profesionales de la salud y cuatro madres. El método que utilizaron estudio cualitativo, revisión integrativa que se realizó en mayo del 2014, en las bases de datos LILACS, BDENF y PubMed por medio de la combinación de las siguientes palabras clave: teoría de la complejidad, pensamiento complejo, Edgar Morin, enfermeros, enfermería, organización, administración, gestión y gerencia. Se incluyeron diez artículos, publicados entre 2004 y 2013. Resultados: se evidenció el crecimiento de la producción científica sobre el pensamiento complejo en la gestión de enfermería y salud. Todos eran artículos originales; siete de abordaje cualitativo.

Los resultados obtenidos en este estudio indican que el pensamiento complejo es un paradigma relacionado con la integralidad, multidisciplinar, atención a las redes de apoyo y articulación de conocimientos. Se ha utilizado como modelo de gestión en organizaciones vivas e impredecibles para promoverlo. organización de sistemas complejos adaptativos. Este estudio contribuye a la aproximación del referencial de complejidad con ellos conceptos de gestión en enfermería y salud, dando mayor visibilidad para futuras publicaciones. Sobre la base de los resultados, se sugiere que es posible la construcción de nuevos modelos de gestión del cuidado para obtener su calidad en sistemas complejos. La construcción de nuevos modelos requiere el esfuerzo de los profesionales en la búsqueda de nuevas formas de interacción y en la búsqueda de nuevas y nuevas formas de afrontamiento prácticas de salud, como posibilidades de transformar su praxis.

La importancia/relevancia fue que los estudios analizados en esta revisión permitieron evaluar la evidencia disponible en la literatura sobre el pensamiento complejo en las publicaciones científicas de 
gestión de enfermería y Salud. Se encontró que la mayoría de las publicaciones se desarrollan en el contexto brasileño y que el pensamiento complejo es emergiendo como un nuevo modelo de atención, tanto en el contexto de atención hospitalaria y primaria, con miras a la integralidad, multidisciplinariedad, redes de atención y articulación desconocimientos. Además, se utilizó como modelo de gestión en organizaciones vivas e impredecibles, con el objetivo de promover la organización de sistemas adaptativos complejos que intentan explicar la dinámica de las organizaciones de una manera más profunda y contextualizada.

Tabla 2 Artículos empíricos

\begin{tabular}{|c|c|c|c|c|}
\hline $\begin{array}{l}\text { Autores, } \\
\text { Título y año }\end{array}$ & $\begin{array}{l}\text { Idioma } \\
\text { original }\end{array}$ & $\begin{array}{c}\text { Objetivo/ } \\
\text { intenciona- } \\
\text { lidad }\end{array}$ & $\begin{array}{l}\text { Situación } \\
\text { Problema }\end{array}$ & Conclusión \\
\hline $\begin{array}{c}\text { Álvarez, S. C. O. } \\
\text { (2019). Importan- } \\
\text { cia del pensa- } \\
\text { miento complejo } \\
\text { del investigador y } \\
\text { la práctica en un } \\
\text { proceso de inves- } \\
\text { tigación científica. } \\
\text { Opcion, 35(90), } \\
\text { 1357-1375. ISSN: } \\
10121587\end{array}$ & Español & $\begin{array}{l}\text { El objetivo } \\
\text { del análisis } \\
\text { es reflexio- } \\
\text { nar sobre la } \\
\text { importancia } \\
\text { del pen- } \\
\text { samiento } \\
\text { complejo del } \\
\text { investigador } \\
\text { y la prác- } \\
\text { tica en un } \\
\text { proceso de } \\
\text { investigación } \\
\text { científica } \\
\text { permitiendo } \\
\text { emprender } \\
\text { el rol profe- } \\
\text { sional como } \\
\text { docente/ } \\
\text { investigador; } \\
\text { desde una } \\
\text { mirada in- } \\
\text { terdisciplina- } \\
\text { ria, transdis- } \\
\text { ciplinaria y } \\
\text { holística. }\end{array}$ & $\begin{array}{l}\text { Nos aproxi- } \\
\text { mamos a una } \\
\text { nueva forma de } \\
\text { pensar y ver la } \\
\text { realidad, intro- } \\
\text { ducir nuevas } \\
\text { ideas, des- } \\
\text { cubrimientos, } \\
\text { conceptos y } \\
\text { teorías que se } \\
\text { tejen alrededor } \\
\text { de un mun- } \\
\text { do diverso y } \\
\text { globalizado ge- } \\
\text { nerando nuevos } \\
\text { paradigmas. De } \\
\text { allí, se entien- } \\
\text { de la comple- } \\
\text { jidad como } \\
\text { un paradigma } \\
\text { que permite la } \\
\text { organización } \\
\text { de un sistema, } \\
\text { generado por } \\
\text { nuevos avances } \\
\text { en ciencia y } \\
\text { tecnología. }\end{array}$ & $\begin{array}{c}\text { El papel del } \\
\text { investigador } \\
\text { social implica la } \\
\text { formación de un } \\
\text { sujeto autóno- } \\
\text { mo, con actitud } \\
\text { crítica, pensa- } \\
\text { miento reflexivo, } \\
\text { investigativo, } \\
\text { creativo, con } \\
\text { capacidad } \\
\text { de análisis y } \\
\text { una decisión } \\
\text { responsable y } \\
\text { libre que los } \\
\text { lleve a construir } \\
\text { y a profundizar } \\
\text { su propio saber } \\
\text { para sí poder } \\
\text { transformar su } \\
\text { propia realidad; } \\
\text { es lo que todo } \\
\text { investigador } \\
\text { debe aportar } \\
\text { al avance de } \\
\text { la ciencia y la } \\
\text { tecnología }\end{array}$ \\
\hline
\end{tabular}


Axpe, M. R. V. (2019). Ciencias de la complejidad vs. Pensamiento complejo. Claves para una lectura crítica del concepto de cientificidad en Carlos Reynoso] Pensamiento, 75(283), 87-106. doi:10.14422/ pen.v75.i283. y2019.004
Este artículo analiza y critica el concepto implícito de cientificidad en el pensamiento de Reynoso y propone Español la necesidad de articular pensamiento filosófico y metodología empírica para comprender la complejidad y sus ciencias
El antropólogo social Carlos Reynoso ha planteado una serie de objeciones al llamado pensamiento complejo, cuyo principal representante es el pensador francés Edgar Morin. Reynoso toma como punto de referencia para realizar la crítica al pensamiento complejo el rigor conceptual e las ciencias de la complejidad
El problema principal para Carlos Reynoso y para todos los que tienen una ideología de tipo cientificista consiste en aceptar que el científico, en un determinado momento, saltará del registro observacional al registro

interpretativoY en ese mismo instante estará utilizando la filosofía como mediación hermenéutica del dato cuantificable; y el lenguaje discursivo, como mediación de la analogía (que se produce como comunicación intersubjetiva). 
Ávila, J. I., E Díaz, L. C. (2019). Emociones en educación matemática: Una mirada con base en el pensamiento complejo https://doi. org/10.1590/2175 623676639.
Este artículo tiene como objetivo presentar una reflexión sobre dos nociones teóricas para el estudio de las emociones en educación matemática: complejidad vivencial y configuración emocional.
Se problematiza

y fundamenta la incorporación de las nociones de complejidad vivencial y configuración emocional. Con estas distinciones se aborda el suceder de configuraciones emocionales de una estudiante de pedagogía en matemáticas, identificando emociones cruciales en su proceso de decisión vocacional al cierre de enseñanza media. 
Rubio, P. (2018).

Aplicación de las teorías de la complejidad a la comprensión del territorio. https:// doi.org/10.3989/ estgeogr.201810

\section{Español}

El primer

objetivo es

comprender

y explicar

el territorio

aplicando

algunas de

las nociones

y princi-

pios de la

complejidad,

y el segundo es argumen-

tar sobre la

utilidad de

los modelos

cualitativos

de síntesis

territorial

para lograr

la intención

anterior $\mathrm{y}$

establecer el segundo
El conocimiento sobre cómo se comporta un territorio no nos permite inferir cómo lo hará otro, con la misma estructura y en las mismas circunstancias. ¿Por qué no siempre deriva idénticas consecuencias de los vínculos entre los componentes del capital territorial? ¿Por Las teorías sobre qué las acciones la complejidad territoriales de proporcionan un desarrollo no trasfondo conson aplicables ceptual apropiade manera indiscriminada? Todos nos hemos hecho estas preguntas alguna vez y nos hemos basado en la complejidad del territorio para responderlas. Sin embargo, creemos que el argumento de la complejidad se ha asumido sin más razón, confundiendo complejidad con complicación 


\begin{tabular}{|c|c|c|c|c|}
\hline $\begin{array}{l}\text { Rodríguez, L. } \\
\text { (2017). Contribu- } \\
\text { ción a la crítica } \\
\text { del pensamiento } \\
\text { complejo de Ed- } \\
\text { gar Morin. Bases } \\
\text { para un programa } \\
\text { de investigación } \\
\text { sobre los para- } \\
\text { digmas. Gazeta } \\
\text { De Antropolo- } \\
\text { gia, 33(2). ISSN: } \\
\text { 02147564 }\end{array}$ & Español & $\begin{array}{c}\text { Elaborar } \\
\text { una crítica } \\
\text { constructiva } \\
\text { a la obra } \\
\text { de Edgar } \\
\text { Morin como } \\
\text { vía estra- } \\
\text { tégica para } \\
\text { regenerar el } \\
\text { pensamiento } \\
\text { complejo. }\end{array}$ & $\begin{array}{c}\text { Superar una de } \\
\text { sus debilidades } \\
\text { más significati- } \\
\text { vas: la carencia } \\
\text { de una metodo- } \\
\text { logía empírica } \\
\text { que permita } \\
\text { desarrollar las } \\
\text { potencialidades } \\
\text { de sus precep- } \\
\text { tos teóricos. }\end{array}$ & $\begin{array}{c}\text { La contribución } \\
\text { de la ciencia } \\
\text { es posible solo } \\
\text { en la medida } \\
\text { en que pueda } \\
\text { ayudarnos a } \\
\text { pensar nuestro } \\
\text { pensamiento, } \\
\text { a construir } \\
\text { observables de } \\
\text { los paradigmas } \\
\text { que mejoren } \\
\text { nuestra auto- } \\
\text { comprensión } \\
\text { sobre los límites } \\
\text { de los sistemas } \\
\text { de pensamiento } \\
\text { donde estamos } \\
\text { atrapados }\end{array}$ \\
\hline
\end{tabular}

El primer artículo de Álvarez, S. C. O. (2019), corresponde a una investigación empírica. Pone a consideración que la interdisciplinariedad se puede ver como un reto en donde los métodos de investigación plantean un nuevo papel y al pensar en el principio de reflexividad se infiere que esta promueve al pensamiento complejo a reflexionar acerca de la investigación científica en una edad contemporánea. Asimismo, extender una invitación que transcienda y promueva la exploración de nuevas formas de trabajo académico e investigativo.

Además, se evidencian los cambios que ha logrado la ciencia al reconocer las nuevas formas de conocimiento, el reconocimiento del papel del investigador, la importancia de las técnicas de investigación y la conceptualización para la emisión de los resultados en investigación. También, se resalta que los pensamientos acerca de un déficit en la investigación han permutado, son aprobados e incluidos en nuestro lenguaje y cultura. Por eso, se hace una invitación a pensar en la interdisciplinariedad como un reto en donde los métodos de investigación esbozan un rol nuevo y en el principio de reflexividad como un recurso para que el pensamiento complejo apunte hacia nuevos horizontes acerca de la investigación científica.

Con los aportes antes señalados, podemos concluir que el proceso investigativo desde la complejidad implica, desarrollar la construcción del conocimiento desde la perspectiva del individuo en el universo, donde lo humano es concebido como un todo. El mundo de hoy presenta desafíos que cada vez son más complejos para nuestra sociedad; los avances, el desarrollo y la revolución de la ciencia y la tecnología nos conducen a ciertos cambios significativos que se ven reflejados en toda la humanidad ; estos cambios nos conduce a tener una visión de nuevos paradigmas basados en la integración de ideas, 
conocimientos, descubrimientos y teorías que representen la realidad para generar nuevas cambios en un sistema complejo entendida como unidad global, es allí, donde aparece la complejidad como resultado de la organización del todo bajo la presión de las infinitas combinaciones de interacciones simultáneas, y que abundan en interrelaciones no-lineales.

El segundo artículo presentado por Axpe, M. R. V. (2019), corresponde a una investigación empírica. Pone a consideración que el antropólogo social Carlos Reynoso ha planteado una serie de objeciones al llamado pensamiento complejo, cuyo principal representante es el pensador francés Edgar Morin. En su obra Complejidad y Caos crítica rigurosamente lo que él considera abusos conceptuales por parte del pensamiento complejo moriniano, aunque también incluye críticas a los planteamientos sobre la complejidad de personalidades de reconocido prestigio científico, tales como el premio Nobel Ilia Prigogine, o los biólogos chilenos Humberto Maturana y Francisco Varela. Así pues, el pensamiento complejo de E. Morin es una especie de epistemología (una nueva forma de mirar la realidad y de entender el propio conocimiento humano de dicha realidad) que funge como pedagogía de una nueva forma de pensamiento: lo que E. Morin denomina el paradigma de la complejidad.

Las ciencias de la complejidad, por el contrario, son desarrollos teóricos con una fuerte base matemática que han nacido en el ámbito de las ciencias naturales y de las ciencias formales. Lo que hace a Carlos Reynoso decantarse por el modelo algorítmico como único modelo válido para entender la complejidad es una deficiente concepción de la ciencia experimental. En alguna ocasión, Reynoso indica que la formalización hace posible las operaciones sin una referencia semántica (que según él es lo que corrompe el discurso científico al introducirse el lenguaje natural).

El problema principal para Carlos Reynoso y para todos los que tienen una ideología de tipo cientificista consiste en aceptar que el científico, en un determinado momento, saltará del registro observacional al registro interpretativo. Y en ese mismo instante estará utilizando la filosofía como mediación hermenéutica del dato cuantificable; y el lenguaje discursivo, como mediación de la analogía (que se produce como comunicación intersubjetiva).

El tercer artículo de Ávila Contreras, J. I., E Díaz Moreno, L. C. (2019), Pone a consideración que la enseñanza y el aprendizaje de las matemáticas suelen identificarse, socialmente, con características de selectividad y racionalidad, tanto para la matemática como para quienes la estudian.

La naturaleza multidimensional de lo humano, la racionalidad florece, emergiendo de modo dinámico, en una relación dialógico-argumentativa entre instancias lógicas, empíricas y vivencias de lo humano.

A diferencia de otras posturas teóricas, como la del dominio afectivo, que analizan a las emociones desde su cristalización, desde la perspectiva expuesta nos interesa situarnos desde el dinamismo del estarse desplegando las emociones. Con este propósito, se ha centrado la atención en aquello que emerge y concurre, retroactivamente, para desvelar complejidades vivenciales 
que configuran a la emoción y, también, en lo que se configura a partir de esas emociones. Se concibe a la configuración emocional como aquella configuración que involucra aspectos vinculados al concurrir y emerger de una emoción, atendiendo a itinerarios que llevan a la emoción a instalarse y desinstalarse en un ahora experiencial de la persona. Esta noción presta atención a esas partes que componen y dan forma a una entidad, involucrando trayectoria y flujo, en los momentos de estarse constituyendo tal entidad. Interesa seguir avanzando en la dirección de comprender, cada vez con mayor profundidad, tramas implícitas que configuran lo vivido y experienciado por los sujetos en sus procesos de formación.

El cuarto artículo de Rubio Terrado, P. (2018), corresponde a una investigación empírica. Pone a consideración que es usual recurrir al argumento de la complejidad al interpretar, por ejemplo, cómo funciona el territorio y qué dificultades suscita comprenderlo, por qué de los enlaces entre sus elementos no derivan los mismos efectos en todos los lugares y en similares circunstancias o por qué lo observado no siempre coincide con lo esperado. Pero la reflexión no se hace desde el paradigma de la complejidad.

Concebir el territorio como un sistema complejo nos coloca en una posición próxima al pensamiento complejo de Morín (1994), quien equipara la complejidad a la capacidad para interconectar las distintas dimensiones de una realidad que está compuesta por elementos interactivos y procesos azarosos. Por territorio entendemos un conjunto de entidades físicas y antrópicas que interactúan. En otras palabras, una globalidad delimitada, localizada, contextualizada y sensible a las variaciones en las condiciones iniciales de sus componentes; en consecuencia, un sistema inestable, emergente, multifuncional y heterogéneo.

Afirmar que el territorio es un sistema complejo pudiera dar lugar a pensar que el azar lo domina todo. Nada más lejos de la realidad. Desde los principios del pensamiento complejo, la comprensión del territorio no lleva a rechazar las certezas en beneficio de la incertidumbre, si bien, siempre estará presente en la prospectiva. Nuestra hipótesis es válida, en tanto en cuanto los principios de la complejidad facilitan comprender el carácter complejo del territorio; es decir, su actividad y los cambios que experimenta, teniendo en cuenta las circunstancias que lo rodean. También se han alcanzado los objetivos. Con relación al primero, se ha justificado que el estudio del hecho geográfico como un sistema complejo proporciona una perspectiva adecuada para la reflexión territorial en sus múltiples dimensiones, elementos, formas, estructuras, procesos, funciones y dinámicas. Adicionalmente, estamos convencidos de que fomenta la perspectiva humanística del discurso geográfico, rompe con el reduccionismo y mecanicismo del análisis positivista, facilita una visión global, permite relajar la dependencia respecto al principio de causalidad eficiente y pone de relieve el carácter del grupo humano como homo accommodaticius. Sobre el segundo, los modelos cualitativos indubitablemente ayudan al análisis del territorio y son fruto del mismo, llevan a un estilo de aprendizaje heurístico y significativo, enfatizan el carácter de la Geografía como ciencia de síntesis de las relaciones entre los elementos del territorio y ayudan a diseñar y prever la dinámica del proyecto de territorio. 
El quinto artículo, Rodríguez Zoya, L. (2017) corresponde a una investigación empírica.

En la obra de Morin hay un descuido mayúsculo de la dimensión técnico-procedimental y operativa de los métodos científicos. El hecho de que la concepción estándar de la metodología de la investigación haya operado una reducción de los problemas metodológicos a cuestiones de índole técnica y procedimental (Rodríguez 2009) es decir, que en ella el método se haya degradado en programa y en aplicación irreflexiva de recetas (Morin 1982), no exime en absoluto al pensamiento complejo de la necesaria tarea de integración crítica y reflexiva de los métodos y las técnicas de investigación en el seno de un método de la complejidad concebido como estrategia de pensamiento.

El pensamiento complejo nos propone volver a pensar lo que somos, nos provoca a pensar cómo pensamos, nos incita a poner en duda nuestro modo de ser histórico, nos convoca a imaginar nuestro futuro como campo incierto de posibilidades, en una palabra, nos alienta a pensar de otro modo. El pensamiento complejo es el nombre de una búsqueda inacabada e inacabable por construir un pensamiento reflexivo que nos permita la autocrítica permanente de nuestro modo de pensar, de decir y de hacer. Solo así podremos alcanzar la "verdadera reforma del modo de pensar" que auguraba Kant (1994: 8).

Es necesario concluir preguntándonos lo siguiente: ¿̇de qué modo la ciencia, en tanto práctica de indagación empírica sobre el mundo, puede ayudarnos a desarrollar un pensamiento complejo? La contribución de la ciencia es posible sólo en la medida en que pueda ayudarnos a pensar nuestro pensamiento, a construir observables de los paradigmas que mejoren nuestra autocomprensión sobre los límites de los sistemas de pensamiento donde estamos atrapados. ¿De qué forma podríamos construir este sistema de metapuntos de vista sobre el pensamiento? El desarrollo de programas de investigación interdisciplinarios sobre los paradigmas es una respuesta posible a este interrogante. Por último, la crítica constructiva a la obra moriniana abre una vía estratégica para regenerar al pensamiento complejo concebido como un ethos científico y filosófico que nos invita a imaginar creativamente la construcción de nuevos posibles

\section{DISCUSIÓN Y CONCLUSIÓN}

En esta breve revisión, en cuanto a estudios "originales" es interesante que de los seis artículos seleccionados por orden de prioridad e importancia todos ellos sean en idioma portugués y que cinco de ellos estén relacionados al área de salud. El único artículo que no es del área de salud es sobre "Educación ciudadana en el marco del pensamiento complejo" siendo del 2016.

Es interesante que la propuesta del Edgard Morin que nace en el campo de la filosofía como un análisis de la sociedad sea asumida en los estudios originales aplicados como modelos de intervención de una mejora en los procesos de salud desde tratamiento sobre el sida en adultos jóvenes (Costa E Meirelles, 2019); la formación en enfermería (Cruz., Araujo., Nascimento., Lima, França \& Oliveira ,2017). o hasta la vivencia de pacientes con trastornos mentales (Ferreira., Brusamarello., Capistrano., Marin., E Maftum ,2017). 
Constatamos que el pensamiento complejo de Morin aún sigue vigente y que ha rebasado el ámbito de lo especulativo a la aplicación práctica en contextos reales, que en nuestros casos son en el área de la salud.

Todo ello hace evidente que la propuesta de pensamiento complejo de Edgard Morin se presenta como un paradigma integrador frente a reduccionismo simplificadores; es asî que el sistema de la complejidad ayuda no solamente entender, cuestionar o analizar la realidad sino a proponer modelos de intervención como lo presentas estos artículos seleccionados.

En referencia a los artículos "empíricos" obtenidos de la base Scopus, nos encontramos que el idioma original base es español con cinco artículos y uno portugués. El área temática de estos cinco artículos es diversa, pero ninguno de ellos aplicados en el campo de la salud a diferencias de los artículos originales, lo cual resulta significativo.

Los temas tratados de estos artículos se basan por un lado en respuesta a los críticos del pensamiento complejo (Axpe,2019); relacionados con las emociones y el ejercicio de la matemática (ÁvilaEDíaz,2019), también relacionados al proceso investigativo ( Álvarez,2019),etc. De igual forma estas propuestas buscan ver lo cotidiano no solamente como un acontecimiento común sino multireferencial donde se le exige al sujeto un pensamiento reflexivo no totalitario por ello encontramos un artículo de estudio donde el pensamiento complejo está ligado al territorio (Rubio,2018). Entonces a mayor comprensión de la complejidad iremos precisando las ideas, realidades o conductas no a manera de compartimentos estancos sino dentro de contextos multidimensionales.
Finalmente, el llegar a vislumbrar un sistema complejo implica un entrenamiento y esfuerzo de trabajo personal, ya que éste de por si no es innato sino aprehendido. El desarrollar el pensamiento complejo no solo beneficia a la persona que los vivencia sino tiene repercusión social, económica, política, etc.

\section{REFERENCIAS}

Álvarez, S. C. O. (2019). Importancia del pensamiento complejo del investigador y la práctica en un proceso de investigación científica. Opcion, 35(90), 1357-1375. ISSN: 10121587

Axpe, M. R. V. (2019). Ciencias de la complejidad vs. Pensamiento complejo. Claves para una lectura crítica del concepto de cientificidad en Carlos Reynosol Pensamiento, 75(283), 87-106. https://doi.org/10.14422/pen.v75. i283.y2019.004

Ávila, J. I., E Díaz, L. C. (2019). Emociones en educación matemática: Una mirada con base en el pensamiento complejo. https://doi.org/10.1590/2175623676639.

Copelli, F. H. S., de Oliveira, R. J. T., de Oliveira, C. M. S., Meirelles, B. H. S., de Mello, A. L. S. F., E Magalhães, A. L. P. (2016). El pensamiento complejo y sus repercusiones en la gestión en enfermería y salud. http://dx.doi. org/10.5294/aqui.2016.16.4.8.

Costa, V. T., \& Meirelles, B. H. S. (2019). Adhesión al tratamiento de los adultos jóvenes que viven 
con VIH/SIDA, según la óptica del pensamiento complejo. https:// doi.org/10.1590/1980-265XTCE-2017-0016

Cruz, R. A., Araujo, E. L., Nascimento, N. M., Lima, R. J., França, J. R., E Oliveira, J. D. (2017). Reflexiones a la luz de la Teoría de la Complejidad y la formación de enfermeras. Revista Brasileira De Enfermagem, 70(1), 236-239. https://doi:10.1590/0034-71672016-0239

Díaz, O. H., E Arboledad, A. P. (2016). Educación ciudadana en el marco del pensamiento complejo. https://doi.org/10.22507/rli. v13n2a12

Ferreira, A. C. Z., Brusamarello, T., Capistrano, F. C., Marin, M. J. S., E Maftum, M. A. (2017). La vivencia del portador de trastorno mental en el uso de psicofármacos en la perspectiva del pensamiento complejo. https://doi.org/10.1590/0104 07072017001000016

Medeiros, S., E Santos, V. (2019). Sistema de medicación y seguridad del paciente a la luz de la teoría de la complejidad. ISSN: 15612961, 08640319

Morin, E. (1993). El método I: La naturaleza de la naturaleza.Cátedra Morin, E. (1994). Introducción al pensamiento complejo.Gedisa

Rodríguez, L. (2017). Contribución a la crítica del pensamiento complejo de Edgar Morin.Bases para un programa de investigación sobre los paradigmas. Gazeta De
Antropología, 33(2). http://hdl. handle.net/10481/49447

Rubio, P. (2018). Aplicación de las teorías de la complejidad a la comprensión del territorio. https:// doi.org/10.3989/estgeogr.201810

\section{Anexo: Estrategia de búsqueda Scopus}

\#1 17,366 documentos TITLE-ABS-KEY ( complejo Y pensante )

\section{\#2 Acceso abierto/2016-2019/ Tipo de documento: Articulo y revisión: 1087 documntos}

TITLE-ABS-KEY ( complejo Y pensante ) Y ( LIMIT-TO ( ACCESSTYPE (OA) ) ) Y ( LIMITTO ( PUBYEAR , 2019 ) O LIMIT-TO ( PUBYEAR, 2018 ) O LIMIT-TO ( PUBYEAR , 2017 ) O LIMITAR A ( PUBYEAR, 2016 ) ) Y ( LIMITAR A ( DOCTYPE, "ar" ) O LIMIT-TO ( DOCTYPE, "re" ) )

\section{AND \\ \#3 Ingles, español, portugués 1,024 documentos}

TITLE-ABS-KEY ( complejo Y pensante ) Y ( LIMIT-TO ( ACCESSTYPE (OA) ) ) Y ( LIMITTO ( PUBYEAR , 2019 ) O LIMIT-TO ( PUBYEAR, 2018 ) O LIMIT-TO ( PUBYEAR, 2017 ) O LIMITAR A ( PUBYEAR, 2016 ) ) Y ( LIMITAR A ( DOCTYPE , "ar" ) O LIMITAR A ( DOCTYPE , "re" ) ) Y ( LIMITAR A ( IDIOMA , "inglés" ) O LIMITAR A ( IDIOMA , "español" ) O LiMITAR A ( IDIOMA , "Portugués" ) ) 


\section{AND}

\#4 ciencias sociales, Artes y humanidades; Psicología 452 documentos

TITLE-ABS-KEY ( complejo Y pensante ) Y ( LIMIT-TO ( ACCESSTYPE (OA) ) ) Y ( LIMITTO ( PUBYEAR , 2019 ) O LIMIT-TO ( PUBYEAR, 2018 ) O LIMIT-TO ( PUBYEAR , 2017 ) O LIMITAR A ( PUBYEAR, 2016 ) ) Y ( LIMITAR A ( DOCTYPE , "ar" ) O LIMITAR A ( DOCTYPE , "re" ) ) Y ( LIMITAR A ( IDIOMA , "Inglés" ) O LIMITAR A ( IDIOMA , "español" ) O LIMITAR A ( IDIOMA , "Portugués" ) ) Y ( LIMITAR -TO ( SUBJAREA , "SOCI" ) OR LIMIT-A ( SUBJAREA , "artes".

Fecha de recepción: 10-07-2020

Fecha de acepción: 27-09-2020 